\title{
Evaluación de la imagen corporal en mujeres con cáncer de mama: una revisión sistemática
}

\section{Body Image of Women with Breast Cancer: A Systematic Review}

\author{
Ma. del Rocío Figueroa Varela ${ }^{\mathrm{a}}$ \\ Universidad de Guadalajara, México \\ ORCID: http://orcid.org/0000-0003-0858-383X \\ María de los Dolores Valadez Sierra \\ Universidad de Guadalajara, México \\ María Elena Rivera Heredia \\ Universidad Michoacana de San Nicolás de Hidalgo, \\ México \\ Roberto Montes Delgado \\ Universidad de Colima, México
}

a Autor de correspondencia. Correo electrónico: marofiva@hotmail.com

Para citar este artículo: Figueroa Varela, M. R., Valadez Sierra, M. D., Rivera Heredia, M. E., \& Montes Delgado, R. (2017). Evaluación de la imagen corporal en mujeres con cáncer de mama: una revisión sistemática. Universitas Psychologica, 16(4), 1-12. http s://doi.org/10.11144/Javeriana.upsy16-4.eicm

\section{RESUMEN}

La imagen corporal (IC) es la representación subjetiva de la percepción, evaluación, valoración y vivencia del cuerpo que proporciona el sentido de sí mismo como una totalidad. Es afectada cuando existen enfermedades oncológicas que implican cambios corporales. En esta revisión sistemática se identificaron instrumentos susceptibles de usarse en Latinoamérica para evaluar la IC de mujeres con cáncer de mama, analizando 34 artículos publicados en inglés y español del periodo 2004-2014 en bases de libre acceso. Instrumentos originados en Europa y Estados Unidos, tales como la Escala de Imagen Corporal de Hopwood y el módulo QLQ BR 23, desarrollado por la Organización Europea para Investigación y Tratamiento en Cáncer (EORTC), con buenas características psicométricas de validez y confiabilidad, se han utilizado en población latinoamericana. Se valora cómo la IC de mujeres con cáncer de mama afecta su sexualidad, las relaciones de pareja, su autoestima, los estilos de afrontamiento y calidad de vida.

Palabras clave

cáncer; evaluación; imagen corporal; instrumentos psicométricos.

\begin{abstract}
Body image is the subjective representation of perception, evaluation, assessment and experience of the body that provides the sense of self as a whole. It is affected when passes through diseases, especially when implies physical changes like oncologic diseases. This systematic review identified instruments to assess body image in women with breast cancer, through the analysis of 34 articles published in English and Spanish during the 2004-2014 period in open access repositories. Instruments originated in Europe and the United States, with the psychometric properties of validity and reliability were found.The Body Image Scale of Hopwood and module QLQ BR 23 of the European Organization for Research and Treatment of Cancer were reported in studies in Latin American population. It is valued
\end{abstract}


how the body image of women with breast cancer affects their sexuality, couple relationships, self-steem, coping styles and quality of life affects their sexuality, relationships, self-esteem, coping styles and quality of life.

Keywords

assessment; body image; cancer; psychometrics.

En América Latina y el Caribe la mayor incidencia de cáncer de mama (CaMa) se presenta en las Bahamas y en Uruguay, donde se estima que aproximadamente 50000 mujeres en esta región morirán en 2030 por este tipo de cáncer (Organización Panamericana de la Salud, 2014). En México, la edad de ocurrencia de este padecimiento se presenta una década antes, en comparación con las mujeres de Estados Unidos de Norteamérica y Europa (51 años), con una prevalencia de 26.1 casos por cada 100 000 mujeres mayores de 25 años (De la VaraSalazar, Suárez-López, Ángeles-Llerenes, TorresMejía, \& Lazcano-Ponce, 2011), estimándose un incremento global de $1.5 \%$ anual de los casos.

Ante un diagnóstico de cáncer, toda la vida y dinámica de la mujer se afecta; su cuerpo e imagen se reconfiguran por las transformaciones a las que se someterá o sometió, debido al tratamiento para combatir el cáncer. La imagen corporal (IC) es una representación subjetiva del cuerpo que agrupa representaciones derivadas de la autopercepción y valoración de ese cuerpo e incluye la interacción de las medidas antropométricas o cuerpo real, la percepción del cuerpo y el cuerpo ideal o aquel donde se definen los estándares de belleza y salud (Sabiston et al., 2010). La imagen corpórea puede ser fuente de satisfacción e insatisfacción así como de preocupación y ansiedad, si no cumple con los ideales sociales, con los que hace una comparación y evaluación cognitiva (Przezdziecki et al., 2013). La IC está relacionada con la identidad, la autoestima, el sentirse atractivo físicamente, el funcionamiento sexual, la actitud hacia la apariencia física, el estado de salud, el funcionamiento físico normal y la percepción del cuerpo como una totalidad (Falk, Reinertsen, Nesvold, Fossa, \& Dahl, 2010).
Se han formulado diferentes constructos teóricos para explicar la satisfacción y valoración positiva o negativa de las características morfológicas asociadas a la IC para determinar lo que una persona piensa, siente y actúa en relación con su cuerpo, reflejando esto en las creencias sobre la importancia e influencia de la apariencia física en la vida y motivándoles, si es el caso, a mantener o conseguir una apariencia física atractiva. Przezdziecki et al. (2013) acotan que la autovalía, entonces, se conecta con el ajuste a los estándares socioculturales y produce tensión y estrés en las mujeres, cuando hay una discrepancia entre el estándar ideal y lo percibido del cuerpo. Por su parte White y Hood (2011) esquematizan cómo la IC se transforma cuando el cuerpo real es atravesado por una neoplasia y se afectan los aspectos de la vida de las mujeres, dadas las intervenciones quirúrgicas necesarias, las cuales son percibidas como una mutilación al cuerpo, cuyas cicatrices les recuerdan permanentemente que han padecido esta enfermedad. Hecho que les genera estrés, vulnerabilidad psicológica y problemas de ajuste, pues las inferencias hechas por las mujeres sobre su corporalidad, las llevan a tener conductas compensatorias, como el retraimiento social y la inhibición emocional; así por ejemplo, una mujer evitará ser abrazada o utilizar prendas como traje de baño, para que no se perciba la ausencia de una o de las dos mamas que fueron removidas por las cirugías.

$\mathrm{Al}$ conocer el diagnóstico de CaMa, la supervivencia es la primera necesidad. El cuerpo se somete a reparación y manipulación para conseguir la salud. Sin embargo, una vez pasado el shock inicial, pueden aparecer otras preocupaciones o áreas problemáticas, como la insatisfacción con la IC (Moreira \& Canavarro, 2010; Moreira et al., 2011; White \& Hood, 2011; Zimmerman et al., 2010).

De acuerdo con Lozano-Arrazol, CupilRodríguez, Alvarado-Aguilar y Granados-García (2011), la distorsión de la IC a nivel perceptivo se produce por una concentración y magnificación de la atención en la zona afectada, pues está en función del defecto percibido. A nivel cognitivo, hay un diálogo interno crítico, culpabilizador y 
devaluativo, centralizado en las pérdidas y en la consecuencia negativas de ello; las creencias disfuncionales sobre su valía les producen insatisfacción, displacer, hostilidad y frustración. A nivel conductual, la vergüenza, el miedo al rechazo de sus parejas y de sus familiares las lleva a comportamientos de evitación y/o compensación, como evitar mirarse al espejo, no ver sus cicatrices (Rincón, Pérez, Borda, \& Martín, 2012) o a formular preguntas constantes para reaseguramiento sobre el estilo de vestimenta: "iNo se nota que me falta un pecho? ¿Está bien colocada la prótesis?”. La IC, entonces, es crucial para el bienestar psicológico y la calidad de vida. Ante la situación de padecer CaMa, es imperante evaluar cómo esta área se modifica y resignifica, al definir que esta imagen es la percepción, evaluación, valoración y vivencia del propio cuerpo, la cual proporciona el sentido de sí mismo como una totalidad.

El objetivo de esta revisión sistemática de artículos científicos fue describir el o los instrumentos reportados para evaluar la IC de mujeres con cáncer de mama, susceptibles de utilizarse en población latinoamericana. Contar con instrumentos adaptados a las pacientes en su contexto sociocultural y con adecuadas propiedades psicométricas para la sensibilidad, confiabilidad y validez de los resultados, pemitirá no solo obtener datos comparables y generalizables con los cuales diseñar adecuadas intervenciones psico-oncológicas, sino también darán cuenta de cómo es vivido el proceso individual por cada mujer que sufre de CaMa, dada la afectación que se tiene en todas las áreas de su vida y, por ende, se podrían prevenir o tratar síntomas relacionados con la IC y su posible afectación a la adherencia al tratamiento.

\section{Método de la revisión de evaluación de imagen corporal en mujeres con cáncer de mama}

Se analizaron bases de datos de libre acceso, a través de la plataforma de EBSCOhost; además, se efectuaron referencias cruzadas con las bases de datos de la Web of Science, Redalyc,
Scielo y Google Scholar. Los términos para la indagación estuvieron relacionados con las palabras claves y operadores boleanos breast cáncer AND body image AND assessment OR measuring instrument. Asimismo, en español, se asociaron para la búsqueda: cáncer de mama AND evaluación AND imagen corporal. Se incluyeron los documentos publicados en el periodo de enero 2004 a junio de 2014 en inglés y en español. En los estudios, debería utilizarse un instrumento de medida estandarizado o especialmente diseñado para la investigación, con propiedades psicométricas reportadas que evaluaran el constructo IC en las fases de detección, tratamiento y rehabilitación en las mujeres con CaMa. Las investigaciones deberían haber sido realizadas con población de la cultura occidental. Además, el acceso al artículo completo debería estar disponible en Internet. Una vez identificados los artículos a través de la búsqueda de bases de datos, con los criterios de inclusión preestablecidos, los documentos se revisaron y analizaron, seleccionando la información relevante y registrando en una base de datos: año de publicación, país en donde se efectuó la investigación, autores, referencia de publicación, instrumentos utilizados, resultados principales y conclusiones.

Los instrumentos de evaluación de la imagen corporal en el cáncer de mama

Se detectaron 77 resúmenes de textos científicos susceptible de revisarse, de los cuales 34 de ellos (44\%) cumplieron con los criterios de inclusión. Se excluyeron 43 (56\%) documentos, por no cumplir con los criterios preestablecidos.

En los artículos analizados, se reportaron diez instrumentos de evaluación para la IC. Tres de ellos fueron los que se utilizaron con mayor frecuencia (Tabla 1), la escala de imagen corporal (BIS), elaborada por Hopwood, Fletcher, Lee y Ghazal en 2001, es el más utilizado en países europeos y angloparlantes, reporta una consistencia interna mediante alpha de Cronbach de 0.93, en un instrumento que evalúa una sola dimensión mediante 10 ítems, cinco de 
los cuales están relacionados con la preocupación general por la IC: autoconsciencia, insatisfacción cuando se está vestida, dificultad para verse desnuda, alejarse de los otros por motivo de la apariencia física e insatisfacción corporal. Cinco ítems están relacionados con la experiencia del cáncer: sentirse menos atractiva físicamente, menor sensación de femineidad, menor atractivo sexual, insatisfacción por cicatrices, creencia de no estar completa. Se reportó su validación al portugués por Moreira, Silva, Marques y Canavarro en 2010. No se encontró reporte específico para la validación en español en el periodo de la búsqueda, aunque ya fue utilizado en España y México (Aguilar, Neri, Mur, \& Gómez, 2013; Juárez \& Landero, 2011; Narváez, Rubiños, Cortés-Funes, Gómez, \& García, 2008). En México, en investigación hecha por Juárez y Landero (2011), donde se aplicó el BIS a mujeres con CaMa, se obtuvo un alfa de Cronbach de 0.84 en el análisis de consistencia interna, asimismo una correlación significativa con la subescala de imagen corporal del QLQ BR23 $\left(r_{s}=0.444, p<0.01\right)$, esto indica que es un instrumento confiable y válido para aplicarse en mujeres mexicanas.

El módulo de calidad de vida en CaMa QLQ BR23 se derivó de la evaluación de la calidad de vida en pacientes oncológicos por el grupo EORTC u Organización Europea para la Investigación y Tratamiento del Cáncer. Consta de 23 ítems con dos escalas, una de funcionalidad en diversas áreas y otra de presencia de síntomas. Está validado en Chile y México y adaptado en Colombia (Cerezo et al., 2012; Irarrázaval et al., 2013; Sánchez, Venegas, Otero, \& Sánchez, 2009), con buenas propiedades psicométricas, resultando un alpha de Cronbach $>0.7$, en sus ítems. Aunque se prioriza la evaluación de la calidad de vida en las investigaciones con este instrumento (Sat-Muñoz et al., 2011).

El Multidemensional Body Self Relations Questionnarie (MBSRQ), mediante 69 reactivos, evalúa siete dimensiones de la $\mathrm{IC}$ y es utilizado en diferentes contextos y poblaciones; fue validado al español en población colombiana, por Botella, Ribas y Benito en 2009, reportando 0.88 de consistencia interna. En contextos oncológicos y en específico en el CaMa, solo se ha utilizado el MBSRQ en Estados Unidos de América y Canadá (Bober, Giobbie-Hurder, Emmons, Winner, \& Partridge, 2013; Sabiston et al., 2010; Stan et al., 2012). En las demás investigaciones revisadas se aplicaron diversas escalas o cuestionarios que, si bien demostraban adecuadas propiedades psicométricas, no se ha generalizado su aplicación en Latinoamérica, para el estudio de la IC en CaMa, como el Body Image after Breast Cancer Questionnarie (BIBCQ), Test de Medición del Grado de Satisfacción Corporal, The Body Shame Subscale of the Experience of Shame Scale (ESS), The Appearance Schemas Inventory Revised (ASI-R), la Escala de Autoconcepto Físico de Tennessee, The Sexual Adjustment and Body Image Scale (SABIS) y la Body Image and Relationship Scale. 


\section{TABLA 1}

Instrumentos para evaluación de la imagen corporal en mujeres con cáncer de mama utilizados con mayor frecuencia en publicaciones analizadas de 2004 a junio 2014

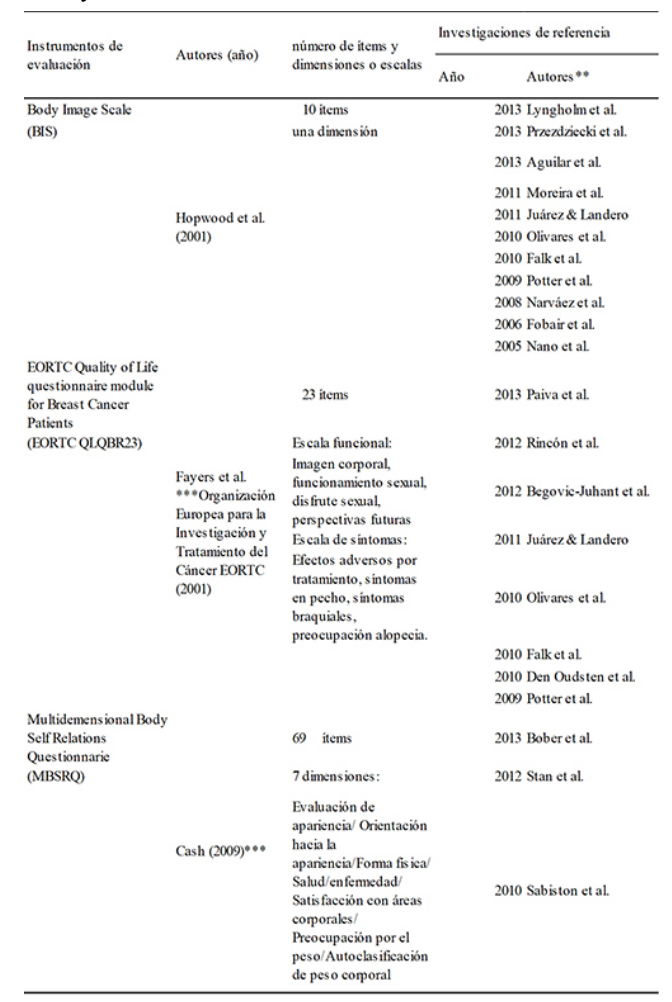

** Nota. Los datos completos de las publicaciones se encuentran en el listado de referencias. Algunas investigaciones utilizaron tanto el BIS como el EORT QLQB 23. Se analizaron 34 artículos. *** Para referencias de estos instrumentos acudir a investigaciones revisadas.

Fuente: elaboración propia.

En las investigaciones, se destaca que esta temática se aborda más en países europeos, como se muestra en la Tabla 2. Estados Unidos de América es el país con más reportes de artículos encontrados. De la población hispanoparlante, España es el país donde más se investiga sobre la IC en mujeres con CaMa. En América Latina, esta área apenas inicia su abordaje. En México, se encuentra el reporte de Aguilar et al. (2013) en coparticipación en una investigación efectuada con España; en ese mismo año, Irarrázaval et al. refieren la investigación hecha en Chile. En Colombia, se ha trabajado esta temática, pero solo como un aspecto por evaluar en la calidad de vida (Sánchez-Pedraza, Sierra-Matamoros, \&
López-Daza, 2012; Sánchez et al., 2009). En Brasil, en 2014 Gonçalves, Tavares, Campana y Cabello dan cuenta de una investigación al respecto.

\section{TABLA 2}

Número de publicaciones por país que reportan instrumentos psicométricos de evaluación para imagen corporal en mujeres con cáncer de mama

\begin{tabular}{lccccr}
\hline Europeos & \multicolumn{3}{c}{ Angloparlantes } & \multicolumn{2}{c}{ Latinoamericanos } \\
\hline Pais & $N$ & Pais & $N$ & País & $n$ \\
\hline Alemania & 1 & Australia & 2 & México & 1 \\
Dinamarca & 1 & Canadá & 2 & Brasil & 1 \\
España & $\mathbf{6}$ & Estados Unidos & $\mathbf{9}$ & Chile & 1 \\
Francia & 1 & Inglaterra & 1 & Subtotal & 3 \\
Holanda & 1 & Subtotal & 14 & $\%$ & 9 \\
Italia & 2 & $\%$ & 41 & & \\
Noruega & 1 & & & & \\
Portugal & 4 & & & & \\
Subtotal & 17 & & & & \\
$\%$ & 50 & & & & \\
\hline
\end{tabular}

** Nota. Investigaciones reportadas en el periodo enero 2004 - junio 2014. Los datos completos de las investigaciones se encuentran en las referencias. Fuente: elaboración propia.

\section{Resultados de la evaluación sobre la imagen corporal y su asociación con otros aspectos psicosociales, en mujeres con cáncer de mama}

A través de los instrumentos utilizados, se hace hincapié en la vivencia en silencio, la autoconciencia e insatisfacción con la imagen corporal de las mujeres con CaMa, pues, ante la magnitud de la enfermedad oncológica que padecen o han padecido, creen que no es importante su imagen, ni su apariencia física, ni las alteraciones en la funcionalidad sexual o sus malestares psicológicos, por ello no manifiestan sus sentimientos y preocupaciones (Dalton et al., 2009; Decker, Pais, Miller, Goulet, \& Fifea, 2012). A continuación, se presentan los resultados sobre la relación de la imagen corporal y diversos aspectos psicosociales, compilados de las diferentes investigaciones analizadas. 


\section{Calidad de vida}

Moreira y Canavarro (2010) consideran a la imagen corporal en las mujeres con CaMa como un componente más en la calidad de vida (CV) y por ello, como una dimensión indicativa y predictiva del distrés que padecen, de acuerdo al tipo de diagnóstico, tratamiento y rehabilitación. Al ser la IC un proceso dinámico, se ha encontrado una satisfacción más baja en los primeros meses después de la mastectomía, pero se mejora con el paso del tiempo, fenómeno parecido a lo que pasa con la edad, pues en etapa de adultez mayor, interesa más la funcionalidad corporal que la apariencia física (Sabiston et al., 2010). La CV en pacientes con CaMa está afectada cuando se está en tratamiento, por los síntomas adversos derivados de las quimio y radioterapias, y es común en la fase de sobrevivencia el miedo a la recurrencia (Moreira et al., 2011). Al sufrir una mastectomía radical, se presentará mayor ansiedad, depresión y peor imagen corporal (Begovic-Juhant, Chmielewski, Iwaugwau, \& Chapman, 2012; Dalton et al., 2009; Zimmerman, Scott, \& Heinrichs, 2010). Por otra parte, reportan mejor CV e imagen corporal las mujeres que tienen cirugías conservadoras (Rosenberg, 2013; Nano et al., 2005) y las que pasan por cirugías reconstructivas (Olivares, Martí, Román, \& Moreno, 2010). Aquellas en las que hay conservación del pezón en la cirugía son las más satisfechas, pues las mujeres consideran esta área de la mama como de crucial importancia (Didier et al., 2008). El tiempo juega un papel adverso en las jóvenes con CaMa y mastectomía, pues después de dos años es cuando la satisfacción corporal parece estar más afectada (Vázquez-Ortiz, Antequera, \& Blanco, 2010; White \& Hood, 2011), dado que el uso de las prótesis, el linfedema o el sobrepeso hacen estragos en esta área (Moreira \& Canavarro, 2010). Si la reconstrucción mamaria se efectúa en la misma cirugía de la mastectomía, hay menos problema en la IC (Rincón et al., 2012). Sobre los resultados de la reconstrucción, son las jóvenes quienes reportan estar más satisfechas con los aspectos cosméticos (Lyngholm, Christiansen,
Damsgaard, \& Overgaard, 2013), pero no se encuentra una clara mejoría en cuanto a la imagen corporal (Rincón et al., 2012) $\mathrm{o}$ al funcionamiento sexual (Bober et al., 2013; Rosenberg et al., 2013), aunque sí mejora su percepción de ser atractivas (Moniz, Fonseca, Fernandes, \& Oliveira, 2011). De manera contradictoria, incluso se ha encontrado que la IC se deteriora más si se efectúa la reconstrucción tiempo después de haberse efectuado la mastectomía radical (Falk et al., 2010), dadas las expectativas estéticas generadas.

Las mujeres más activas físicamente desde antes de la enfermedad, tenderán ha tener mejor funcionamiento social que aquellas más pasivas (Hormes et al., 2008), sin embargo, estas actividades aunque les permitan mejorar su apreciación de CV no les mejora su imagen corporal (Stan et al., 2012).

\section{Autoestima y el ser "incompletas"}

Se encontró asociación de la tendencia a basar el autoconcepto y autoestima en el atractivo físico con presencia de depresión, en la fase de recuperación de CaMa (Moreira \& Canavarro, 2010). Un factor de protección para la calidad de vida, y en especial para la disminución de los síntomas depresivos, es la construccion de una relación de pareja satisfactoria (Zimmerman et al., 2010) que las apoyará frente a los tratamientos. Los esfuerzos por mejorar la apariencia o sentirse atractivas son un factor de protección psicosocial para afrontar el CaMa, pero si hay creencias de que la apariencia física define categóricamente su autoestima o autoconcepto, por el contrario, se vuelve la imagen un factor de vulnerabilidad psicológica (Moreira \& Canavarro, 2010), como ocurre en el caso de las mujeres jóvenes con CaMa, pues las mujeres de mayor edad presentan mejor aceptación de sí mismas (Hormes et al., 2008; Przezdziecki et al., 2013). Incluso, las mujeres, aunque tengan tratamientos de belleza específicos durante su tratamiento para mejorar IC, no reportan mejoría en sus niveles de estrés (Quintard \& Lakdja, 2008). La imagen corporal 
y la autoestima presentan menos problemas en las mujeres con cirugías conservadoras de mama (Sebastian, Manos, Bueno, \& Mateos, 2007). Sin embargo, mediante los instrumentos de evaluación se detecta que las mujeres sienten vergüenza por su cuerpo (Biglia et al., 2010), aun cuando se someten a cirugía reconstructiva (Frierson, Thiel, \& Andersen, 2006; Moreira \& Canavarro, 2010), así como miedo a mostrar su cuerpo, al sentir devaluadas su atractividad y femineidad (Fobair, 2006) lo que las hace dudar de su identidad de género, al aducir que son menos femeninas cuando pierden la(s) mama(s).

Así, la imagen corporal de las mujeres es como si estuviera fracturada, y la mama o mamas que se retiran son percibidas como una pieza faltante en su representación subjetiva, como un rompecabezas incompleto de sí mismas (Moreira, Silva, \& Canavarro, 2010). Al presentar pensamientos intrusivos disfuncionales sobre la propia valía, la falta de un seno o presencia de una cicatriz, lo asumen como la totalidad de la apariencia y de sí mismas (Frierson et al., 2006). En estudio comparativo entre españolas y mexicanas, estas últimas refieren sentirse más incompletas (Aguilar et al., 2013), esto indica presencia de factores socioculturales asociados a esta percepción de "ser incompletas", en México.

\section{Estilos de afrontamiento}

Aquellas mujeres con CaMa y con afrontamiento optimista puntúan mejor en su satisfacción con la IC y presentan mejor adaptación psicológica a su enfermedad, así como también a mejores índices de sobrevivencia y calidad de vida (Barsotti \& Meloni, 2011; Juárez \& Landero, 2011). La religiosidad, estrategia de afrontamiento que disminuye los síntomas aversivos derivados de los tratamientos, sobre todo al inicio del diagnóstico y durante la quimioterapia (Paiva et al., 2013), presenta una relación positiva con la funcionalidad física.

\section{Relaciones de pareja y funcionalidad sexual}

Al ser las mamas una zona erógena, la funcionalidad sexual se compromete ante el CaMa (Moreira et al., 2011). El disfrute de la relación sexual no está en relación al tipo de cirugía mamaria, sino por la valoración o evaluación de la IC y su preocupación por seguir siendo atractivas sexualmente, especialmente si se consideran estar bajo el estigma de padecer cáncer, aun incluso después de haber terminado el tratamiento aversivo (Gonçalves et al., 2014; Lyngholm et al., 2013; Rincón et al., 2012). En general, al inicio puede haber dificultades en la sexualidad de las mujeres con CaMa (Barsotti \& Meloni, 2011; Moreira et al., 2011), la pérdida del cabello y cambios de peso les hace sentirse menos atractivas (Vázquez-Ortiz et al., 2010) y con menos deseo sexual (Fobair et al., 2006), pero también ellas manifiestan que cambian sus hábitos para vestirse, al evitar mostrarse desnudas (Lyngholm et al., 2013). Su inquietud por la desfiguración física y la alteración de la intimidad sexual las lleva a creerse menos valoradas, con miedo al rechazo de su pareja, quejándose de que les dejan de acariciar el pecho por ellos (VázquezOrtiz et al., 2010).

El padecer CaMa puede recrudecer problemática previamente existente en la funcionalidad sexual (Bober et al., 2013). Las relaciones de pareja, si estaban deterioradas o tibias, sufrirán mayor presión y terminan en abandono o ruptura de la relación, al no soportar la presión. Por el contrario, si se tiene buena relación de pareja, los conflictos se subsanan, una vez pasado el tratamiento (Den Oudsten, Van Heck, Van der Steeg, Roukema, \& De Vries, 2010; Moreira et al., 2011; Zimmerman et al., 2010). La satisfacción marital que reflejen las parejas de las mujeres con CaMa, apoya su imagen corporal, de tal suerte que si la pareja está insatisfecha con su relación, impactará también en cómo se autoacepten las mujeres.

Las jóvenes mujeres con CaMa reportan mejor disfrute sexual durante las diversas fases de la enfermedad (Olivares et al., 2010) y las de mayor edad, menos interés en la sexualidad. Las 
mujeres que han desarrollado problemas como el linfedema son más proclives a presentar mayor problemática en la sexualidad (Rincón et al., 2012).

En las mujeres latinas, se encuentra la satisfacción con la IC sin alta relación en la funcionalidad sexual de las mujeres; al ser la sexualidad aún un tabú, las pacientes no informan tener problemas en esta área: "ya no sirvo para eso", "debo estar agradecida con estar viva, lo demás no importa”. Así también encontraron la preocupación sobre su cuerpo más referida a la enfermedad del cáncer en sí que a su valoración de IC (Fobair et al., 2006; Juárez \& Landero, 2011). Debido a esta disparidad de perspectivas y a la fuerte relación de la imagen corporal y la funcionalidad sexual de las mujeres al momento de enfrentar el CaMa, se sugiere elaborar intervenciones especiales que exploren y apoyen estos aspectos (Hormes et al., 2008), aunque para las mujeres y sus parejas sea embarazoso tocar este tópico, o bien se tema causar disgusto o molestia al otro, si se discute sobre el cáncer y el impacto en su relación de pareja (Decker et al., 2012).

\section{Alcances y limitaciones de la revisión}

En conclusión, la revisión sistemática efectuada sobre los instrumentos de evaluación de IC en el cáncer de mama y su asociación con diferentes aspectos psicosociales y de la vida de la mujer, refleja que en Latinoamérica es un campo que no ha sido lo suficientemente explorado. Los instrumentos que han sido adaptados con adecuadas propiedades psicométricas en Latinoamérica son la Escala de Imagen Corporal de Hopwood (BIS) y el módulo de QLQ BR 23 desarrollado por el grupo de investigación en cáncer EORTC.

Si las mujeres consideran la apariencia física como aspecto muy importante en su vida, tendrán más alto nivel de estrés ante el CaMa (Baxter et al., 2006) y, como los instrumentos para detectar niveles de calidad de vida no siempre son sensibles a todos los aspectos (Potter, Thompson, Greenwood, Hopwood, \& Winters,
2009), se debe evaluar la IC a través instrumentos específicos, a fin de detectar estas afectaciones que pueden ser desapercibidas por el equipo médico, y crear alternativas de intervención psicooncológica para mejorar la calidad de vida de las pacientes. Especialmente, se debe seguir investigando en las mujeres latinoamericanas aspectos relacionados con la sexualidad y las relaciones de pareja, dado que se reflejan aún tabúes sobre esta problemática.

Esta revisión tuvo algunas limitaciones como: el periodo determinado de la búsqueda, los idiomas seleccionados y las palabras claves utilizadas, lo que pudo haber dejado sin incorporar documentos pertinentes. Sin embargo, da cuenta de la necesidad de reconocer cuáles son los instrumentos validados en población latinoamericana para evaluar la imagen corporal. Utilizar instrumentos válidos y confiables coadyuvará en la obtención de datos objetivos que permitan, en la investigación y en la práctica clínica, contar con información relevante acerca del bienestar y salud mental de las mujeres con CaMa, así como el impacto de cualquier intervención y su incidencia en la adherencia terapéutica. Esto facilitará hacer los ajustes en el tratamiento, intervenciones y asesorías psicooncológicas, así como la interacción del equipo con un enfoque holístico, donde no se vea a un cuerpo que padece cáncer de mama, sino a una mujer en proceso de reconfiguración de su corporalidad, cuyo cuerpo es portador en sí, del cáncer.

\section{Agradecimientos}

Este trabajo se efectuó con el apoyo del del Consejo Nacional de Ciencia y Tecnología (CONACYT) dentro del Doctorado Interinstitucional de Psicología (DIP), en México.

\section{Referencias}

Aguilar, M. J., Neri, M., Mur, N., \& Gómez, E. (2013). Influencia del contexto social en la percepción de la imagen corporal de las 
mujeres intervenidas de cáncer de mama. Nutrición Hospitalaria, 28(5), 1453-1457. https://doi.org/10.3305/nh.2013.28.5.6517

Barsotti Santosi, D., \& Meloni Vieirall, E. (2011). Imagem corporal de mulheres com câncer de mama: uma revisão sistemática da literatura. Ciencia y Saúde Colectiva, 16(5), 2511-2522. https://doi.org/10.1590/ S1413-81232011000500021

Baxter, N. N., Goodwin, P. J., Mcleod, R. S., Dion, R., Devins, G., \& Bombardier, C. (2006). Reliability and validity of the Body Image after Breast Cancer Questionnaire. The Breast Journal, 12(3), 221-232.

Begovic-Juhant,A., Chmielewski, A., Iwaugwau, S. \& Chapman L.A. (2012). Impact of body image on depression and quality of life among women with breast cancer. Journal of Psychosocial Oncology, 30(4), 446-460. doi: 10.1080/07347332.2012.684856

Biglia, N., Moggio, G., Peano, E., Sgandurra, P., Ponzone, R., Nappi, R., \& Sismondi, P. (2010). Effects of surgical and adjuvant therapies for breast cancer on sexuality, cognitive functions, and body weight. Journal of Sexual Medicine, 7(5), 1891-1900. https://doi.org/10.1111/ j.1743-6109.2010.01725.x

Bober, S., Giobbie-Hurder, A., Emmons, M., Winner, E., \& Partridge, A. (2013). Psychosexual functioning and body image following a diagnosis of ductal carcinoma in situ. Journal of Sexual Medicine, 10(2), 370-377. https://doi.org/10.1111/ j.1743-6109.2012.02852.x

Botella, L., Ribas, E., \& Benito, J. (2009). Evaluación psicométrica de la imagen corporal: validación de la versión española del multidimensional Body Self Relations Questionnaire (MBSRQ). Revista Argentina de Clínica Psicológica, 18(3), 253-264.

Cerezo, O., Oñate-Ocaña, L. F., Arrieta-Joffe, P., González-Lara, F., García-Pasquel, M. J., Bargalló-Rocha, E, ... Villar-Compte, D. (2012). Validation of the MexicanSpanish version of the EORTEC QLQ-C30 and BR23 questionnaires to assess healthrelated quality of life in Mexican women with breast cancer. Europe Journal of Cancer Care, 21(5), 684-691. https://doi.org/ 10.1111/j.1365-2354.2012.01336.x

Dalton, J., Naisbitt, V., Classen, C., Grumann, M., Gronskaya, O., Zarcone, J., ... Spiegel, D. (2009). Sexual Adjustment and Body Image Scale (SABIS): A new measure for breast cancer patients. The Breast Journal, 15(3), 287-290. https://doi.org/10.1111/ j.1524-4741.2009.00718.x

De La Vara-Salazar, E., Suárez-López, L., Angeles-Llerenes, A., Torres-Mejía, G., \& Lazcano-Ponce, E. (2011). Tendencias de la mortalidad por cáncer de mama en México, 1980-2009. Salud Pública de México, 53(5), 385-393.

Decker, C., Pais, S., Miller, K., Goulet, R., \& Fifea, B. (2012). A brief intervention to minimize psychosexual morbidity in dyads coping with breast cancer. Oncology Nursing Forum, 39(2), 176-185. https:// doi.org/10.1188/12.ONF.176-185

Den Oudsten, B. L., Van Heck, G. L., Van der Steeg, A. F., Roukema, J. A., \& De Vries, J. (2010). Clinical factors are not the best predictors of quality of sexual life and sexual function. Psychooncology, 19(6), 646-656. https://doi.org/10.1002/pon.1610

Didier, F., Radice, D., Gandini S., Bedolis, R., Rotmensz, N., Maldifassi, A., ... Petit, J. Y. (2008). Does nipple preservation in mastectomy improve satisfaction with cosmetic results, psychological adjustment, body image and sexuality? Breast Cancer Research and Treatment, 118(3), 623-633. https://doi.org/10.1007/ s10549-008-0238-4

Falk Dahl, C., Reinertsen, K. V., Nesvold, I. -L., Fossa, S. D., \& Dahl, A. A. (2010). A study of body image in long-term breast cancer survivors. Cancer, 116(15), 3549-3557. https:// doi.org/10.1002/cncr.25251, 3549-3557

Fobair, P., Stewart, S., Chang, S., D'Onofrio, C., Banks, P., \& Bloom, J. (2006). Body image and sexual problems in young women with breast cancer. Psychooncology, 15(7), 579-594. https://doi.org/10.1002/pon.991 
Frierson, G., Thiel, D., \& Andersen, B. (2006). Body change stress for women with breast cancer: The BreastImpact of Treatment Scale. Annals of Behavourial Medicine, 32(1), 77-81. https:// doi.org/10.1207/s15324796abm3201_9

Gonçalves, C., Tavares, M. C., Campana, A. N., \& Cabello, C. (2014). Validation of the instrument "Body image after breast cancer" in Brazil. Motriz: Revista de Educação Física, 20(1), 8-15. https://doi.org/10.1590/ S1980-65742014000100002

Hopwood, P., Fletcher, I., Lee, A., \& Al Ghazal, S. (2001). A body image scale for use with cancer patients. European Journal of Cancer, 37(2), 189-197.

Hormes, J., Lytle, L., Gross, C., Ahmed, R., Troxel, A., \& Schmitz, K. (2008). The Body Image and Relationships Scale: Development and validation of a measure of body image in female breast cancer survivors. Journal of Clinical Oncology, 26(8), 1269-1274. https://doi.org/10.1200/ JCO.2007.14.2661

Irarrázaval, M. E., Rodríguez, P., Fasce, G., Silva, F., Waintrub, H., Torres, C., ... Marín, L. (2013). Calidad de vida en cáncer de mama: validación del cuestionario BR23 en Chile. Revista Médica de Chile, 141, 723-734. https://doi.org/10.4067/ S0034-98872013000600006

Juárez, D.M. \& Landero, R. (2011). Imagen corporal, funcionamiento sexual, autoestima y optimismo en mujeres con cáncer de mama. Nova Scientia, 4(7), 17-34.

Lyngholm, C. D., Christiansen, P. M., Damsgaard, T. E., \& Overgaard, J. (2013). Long-term follow-up of late morbidity, cosmetic outcome and body image after breast conserving therapy. A study from the Danish Breast Cancer Cooperative Group (DBCG). Acta Oncologica, 52(2), 259-269. https:// doi.org/10.3109/0284186X.2012.744469

Lozano-Arrazola, A., Cupil-Rodríguez, A. L., Alvarado-Aguilar, S., \& Granados-García, M. (2011). Impacto psicológico en la imagen corporal de pacientes con cáncer de cabeza y cuello sometidos a cirugías deformantes. Gaceta Mexicana de Oncología, 10(3), 156-161.

Moniz, P. A., Fernandes, A.M., \& Oliveira, L. (2011). Implicações da mastectomia na sexualidade e imagem corporal da mulher e resposta da enfermagem perioperatória. Revista de Enfermagem Referência, serIII(5), 163-171. https:// doi.org/10.12707/RIII1148

Moreira, H., \& Canavarro, M. C. (2010). A longitudinal study about the body image and psychosocial adjustment of breast cancer patients during the course of the disease. European Journal of Oncology Nursing, 14(4), 263-270. https:// doi.org/10.1016/j.ejon.2010.04.001

Moreira, H., Crespo, C., Paredes, T., Silva, S., Canavarro, M. C., \& Dattilio, F. M. (2011). Marital relationship, body image and psychological quality of life among breast cancer patients: The moderating role of the disease's phases. Contemporary Family Therapy: An International Journal, 33(2), 161-178. https://doi.org/10.1007/ s10591-011-9149-3

Moreira, H., Silva, S., \& Canavarro, M. C. (2010). The role of appearance investment in the adjustment of women with breast cancer. Psychooncology, 19(9), 959-966. https://doi.org/10.1002/pon.1647

Moreira, H., Silva, S., Marques, A., \& Canavarro, M. C. (2010). The Portuguese version of the Body Image Scale (BIS): Psychometric properties in a sample of breast cancer patients. European Journal of Oncology Nursing, 14(2), 111-118. https:// doi.org/10.1016/j.ejon.2009.09.007

Nano, M. T., Gill, P. G., Kollias, J., Bochner, M. A., Malycha, P., \& Winefield, H. R. (2005). Psychological impact and cosmetic outcome of surgical breast cancer strategies. ANZ Journal of Surgery, 75(11), 940-947. https://doi.org/ 10.1111/ j.1445-2197.2005.03517.x

Narváez, A., Rubiños, C., Cortés-Funes, F., Gómez, R., \& García, A. (2008). Valoración de la eficacia de una 
terapia grupal cognitivo conductual en la imagen corporal, autoestima, sexualidad y malestar emocional (ansiedad y depresión) en pacientes de cáncer de mama. Psicooncología, 5(1), 93-102.

Olivares, M. E., Martí, M. D., Román, J. M., \& Moreno, A. (2010). Valoración biopsicosocial en pacientes con patología mamaria oncológica quirúrgica. Psicooncología, 7(1), 81-97.

Organización Panamericana de la Salud. (2014). Cáncer de mama en las Américas. Recuperado de http://www.paho.org/hq/index.php?opti on $=$ com_docman\&task $=$ doc_view\&gid $=17926$ \& Itemid

Paiva, C. E., Paiva, B. S., de Castro, R. A., Souza, C. de P., de Paiva Maia, Y. C., Ayres, J. A., \& Michelin, O. C. (2013). A pilot study addressing the impact of religious practice on quality of life of breast cancer patients during chemotherapy. Journal of Religion Health, 52(1), 184-193. https:// doi.org/10.1007/s10943-011-9468-6

Potter, S., Thompson, H., Greenwood, R., Hopwood, P., \& Winters, Z. (2009). Healthrelated quality of life assessment after breast reconstruction. British Journal of Surgery, 96(6), 613-620. https://doi.org/10.1002/ bjs. 6605

Przezdziecki, A., Sherman, K., Baillie, A., Taylor, A., Foley, E., \& Stalgis-Bilinski, K. (2013). My changed body: Breast cancer, body image, distress and self-compassion. Psychooncology, 22(8), 1872-1879. https:// doi.org/10.1002/pon.3230

Quintard, B., \& Lakdja, F. (2008). Assessing the effect of beauty treatments on psychological distress, body image, and coping: A longitudinal study of patients undergoing surgical procedures for breast cancer. Psychooncology, 17(10), 1032-1038. https:// doi.org/10.1002/pon.1321

Rincón, M. E., Pérez, M. A., Borda, M., \& Martín, A. (2012). Impacto de la reconstrucción mamaria sobre la autoestima y la imagen corporal en pacientes con cáncer de mama. Universitas Psychologica, 11(1), 25-41.

Rosenberg, S., Tamimi, R., Gelber, S., Ruddy, K., Kareakoglow, S., Borges V., ... Partridge, A. (2013). Body image in recently diagnosed young women with early breast cancer. Psychooncology, 22(8), 1849-1855. https:// doi.org/10.1002/pon.3221

Sabiston, C., Rusticus, S., Brunet, J., McDonough, M., Hadd, V., Hubley, A., \& Crocker P. (2010). Invariance test of the multidimensional body self-relations questionnaire: Do women with breast cancer interpret this measure differently? Quality of Life Research, 19(8), 1171-1180. https://doi.org/10.1007/s11136-010-9680

Sánchez, R., Venegas, M., Otero, J., \& Sánchez, O. (2009). Adaptación transcultural de dos escalas para medir la calidad de vida en pacientes con cáncer en Colombia: EORTC QLQ-C30 y QLQBR23. Revista Colombiana de Cancerología, 13(4), 205-212.

Sánchez-Pedraza, R., Sierra-Matamoros, F. B., \& López-Daza, D. F. (2012). Validación colombiana de la escala FACT-B para medir la calidad de vida de pacientes con cáncer de mama. Revista Colombiana de Obstetricia y Ginecología, 63(3), 196-206.

Sat-Muñoz, D., Contreras-Hernández, I., Balderas-Peña, L. M., Hernández-Chávez, G. A., Solano-Murillo, P., MariscalRamírez, I., ... Morgan Villela, G. (2011). Calidad de vida en mujeres mexicanas con cáncer de mama en diferentes etapas clínicas y su asociación con características socio-demográficas, estados co-mórbidos y características del proceso de atención en el Instituto Mexicano del Seguro Social. Value in Health, 4(5), 133-136. https:// doi.org/10.1016/j.jval.2011.05.027

Sebastián J., Manos D., Bueno, M. J., \& Mateos, N. (2007). Imagen corporal y autoestima en mujeres con cáncer de mama participantes en un programa de intervención psicosocial. Clínica y Salud, 18(2), 137-161. 
Stan, D., Rausch, S., Sundt, K., Cheville, A., Youdas, J., Boughey, J., ... Pruthi, S. (2012). Pilates for breast cancer survivors: Impact on physical parameters and quality of life after mastectomy. Clinical Journal of Oncology Nursing, 16(2) 131-141. https:// doi.org/10.1188/12.CJON.131-141

Vázquez-Ortiz, J., Antequera, R., \& Blanco Picabia, A. (2010). Ajuste sexual e imagen corporal en mujeres mastectomizadas por cáncer de mama. Psicooncología, 7(2-3), 433-451.

White, C., \& Hood, C. (2011). Body image issues in oncology. En T. Cash \& L. Smolak (Eds.), Body image. A handbook of science, practice and prevention (pp. 333-341). Nueva York: The Guilford Press.

Zimmerman, T., Scott, J., \& Heinrichs, N. (2010). Individual and dyadic predictors of body image in women with breast cancer. Psychooncology, 19(10), 1061-1068. https:// doi.org/10.1002/pon.1660

\section{Notas}

* Artículo de revision. 